\title{
Improved Wavelet Threshold for Gray Scale Image Denoising
}

\author{
Liying Yuan, Junfeng $\mathrm{Wu}$ and Shuangquan Li \\ School of Automation, Harbin University of Science and Technology, China \\ yly@hrbust.edu.cn
}

\begin{abstract}
During the image acquisition and communication the image is corrupted by noise. This is a classical problem in the field of signal or image processing. A new compromise threshold method that improves performance is presented, based on the standard compromise threshold method, yet more flexible and easier to treat mathematically. The improved adaptive wavelet compromise method avoids the discontinuity of the hard-threshold method, at the same time increase the correlation judgment process, improve image details loss problem. Simulation results show that the improved method is better at the adaptability, at the same time increase the correlation judgment process, improve image details loss problem.
\end{abstract}

Keywords: wavelet transforms, threshold denoising, correlation, adaptive

\section{Introduction}

Digital images are often degraded by noise in the acquisition and transmission phase. The goal of image denoising is to recover the true original image from such a distorted noisy copy. There are two basic approaches to image denoising, spatial filtering methods and transform filtering methods. In transform domain, wavelets give a superior performance in image denoising due to its properties such as scarcity, energy compaction and multire solution structure. So, the focus was shifted from the spatial and Fourier domain to the wavelet transform domain. In wavelet transform, the small coefficients are more likely due to noise and large coefficients are more likely due to important feature of the image [1,2].

The basic thought of wavelet transform is using the same function by expanding and shifting to approach the original signal. The wavelet coefficients carry the time-frequency information in certain areas. It has good local characteristics both in time domain and frequency domain. It can maintain the fine structure of the original images in various resolutions and it is convenient to combine with human vision characteristics.

Compared with the orthogonal wavelet, bi-orthogonal wavelet has more obvious superiority in image processing because it balances the orthogonality and symmetry. In addition, the reconstructing signal of biorthogonal wavelet transform is suitable to embed watermark for its balance [3].

A vast literature has emerged recently on signal denoising using wavelet theory, in the setting of additive white Gaussian noise. Since the early 1990s, the wavelet theory has been well developed and widely introduced into many fields of applications, including statistics estimation, density estimation, and solution of partial differential equations, image compression and signal processing. In 1992, Donoho and Johnstone presented a method named wavelet shrinkage, and showed its obvious efficiency on signal denoising and inverse problem solving [4]. 
Since the threshold plays a key role in this appealing method, variant methods appeared later to set an appropriate threshold [5, 6]. Donoho and Johnstone found that in the criterion under which the universal threshold is nearly optimal, the smoothness restriction is probably the cause for over-erasing wavelet coefficients. Therefore, they removed the restriction and gave what is known as the Stein's Unbiased Risk Estimate (SURE) threshold, which minimizes SURE, an estimate of MSE risk [7].

The gray-scale image is composed of three channels by red, green, blue, but is displayed in grayscale. This paper focuses on the gray-scale image de-noising method. Traditional denoising method the denoising will affect the details of the portion of the image. In order to keep the details of the image, using wavelet transform method for image denoising, usually threshold denoising method and scale denoising method are based on wavelet transform theory. [8-10] proposed a wavelet thresholding method, can effectively remove noise, simple to implement, has been widely used.

However, these method still has some drawbacks, such as lack of self-adaptive, and not be able to be removed a small amount of noise. In this paper, an adaptive threshold denoising method based on biorthogonal wavelet transforms was provided to improve these deficiencies, which is a high de-noising, edge detail intact preservation of the de-noising method.

This paper is organized as the following. In Section 2, we introduce the wavelet analysis and threshold denoising. In Section 3, we introduce new threshold of change associated with the decomposition level in detail and algorithm. In Section 4, we will apply the algorithm into image denoising. Finally, we show that the new algorithm can achieve state-of-the-art denoising performance.

\section{Wavelet Analysis and Threshold Denoising}

Consider the following additive noise model in time domain

$$
x(i)=s(i)+\varepsilon(i)
$$

Where the signal $\mathrm{s}$ is corrupted by the zero-mean white Gaussian noise process $\varepsilon \sim$ $N\left(0, \sigma^{2}\right)$, resulting in $\mathrm{x}$, the observed, i.e., noisy signal. Throughout this text, $A(i)$ is often used to stand for the ith element of a given sequence A. By the linearity of wavelet transformation, there is a regression model in the wavelet domain

$$
w_{x}(i)=w_{s}(i)+w_{n}(i)
$$

Where $w_{x}$ is the wavelet transform of $\mathrm{x}$, while $w_{s}$ and $w_{n}$ are those corresponding to $\mathrm{s}$ and $\varepsilon$. The elements of $w_{x}, w_{s}$ and $w_{n}$ will be termed as data coefficients (noisy coefficients), signal coefficients (noiseless coefficients), and noise coefficients, respectively. The term denoised coefficients is used to refer to the estimate of the noiseless coefficient from the corresponding noisy coefficient while significant noisy coefficient is also introduced ere to represent a noisy coefficient where signal dominates.

Threshold denoising image decomposition wavelet coefficients by setting the threshold comparison and processing to get the new wavelet coefficients, we can get image denoising by reconstruction of wavelet. There are hard threshold and soft threshold method commonly used in threshold denoising method [10]. Hard threshold and soft threshold are 


$$
\begin{aligned}
& x_{\delta}= \begin{cases}x & |x| \geq \delta \\
0 & |x|<\delta\end{cases} \\
& x_{\delta}=\left\{\begin{array}{cc}
\operatorname{sgn}(x)(|x|-\delta), & |x| \geq \delta \\
0, & |x|<\delta
\end{array}\right.
\end{aligned}
$$

Where $\delta$ is threshold, $x$ is Wavelet coefficients, $x_{\delta}$ is Wavelet coefficients after threshold processing.

The universal threshold is

$$
\delta=\sigma \sqrt{2 \log n}
$$

Where $\sigma$ is the Standard deviation of the noise? The noise variance $\sigma^{2}$ needs to be estimated first. In some situations, it may be possible to measure $\sigma^{2}$ based on information other than the corrupted image. If such is not the case, it is estimated from the subband $\mathrm{HH} 1$ by the robust median estimator

$$
\hat{\sigma}=\operatorname{Median}(|x|) / 0.6745
$$

For a given threshold, soft thresholding has smaller variance, however, higher bias than hard thresholding, especially for very large wavelet coefficients. If the coefficients distribute densely close to the threshold, hard thresholding will show large variance and bias. For soft thresholding, smaller error often happens when the coefficient is close to zero. In general, choose soft thresholding for smoothness while hard thresholding for lower error.

The experiments show that the hard threshold de-noising and soft threshold cannot achieve satisfactory results. Hard threshold method can retain the details of the edge of the image, but because the function is not continuous cause ringing and the generation of the pseudo-Gibbs phenomena. The soft threshold handling can improve the ringing and pseudo-Gibbs phenomena, but because there is a deviation, and blurring the image.

Soft and hard threshold compromise processing method is proposed in [11]

$$
x_{\delta}=\left\{\begin{array}{cc}
\operatorname{sgn}(x)(|x|-\alpha \cdot \delta), & |x| \geq \delta \\
0, & |x|<\delta
\end{array}\right.
$$

Where $\alpha$ Ranges from 0 to 1, drawn between the soft and hard threshold can improve the lack of soft and hard threshold de-noising. But here $\alpha$ is a constant, the change cannot be performed based on the coefficients of the transform, i.e., does not have a self-adaptive, and therefore there are still some defects.

\section{Improved Adaptive Threshold Denoising}

\subsection{Threshold Selection}

The experiments show that the first level of the noise figure wavelet sub-band coefficients of variance is almost equal to the noise variance. With the increase of decomposition levels, the noise variance coefficient becomes smaller and smaller [12].

Donoho selected a single threshold is given as

$$
\delta=\frac{\log _{2}(1+2 \sqrt{N})}{J+Z} A
$$


Where, $N$ is the noise power of the default; $\mathrm{J}$ taken as the maximum size of the Degree; $Z$ is a constant, taken as 2; A is extreme point of the maximum amplitude.

Unlike the universal threshold, which depends only on the sample size of the signal and the variance of the noise, these thresholds are more adaptive to the features of the signal and provide better results. Better results can be attained if the local features of the signal are considered and a more adaptive threshold is given.

To the relationship is reflected in the threshold formula, the formula (4) improvements, so that the new threshold of change associated with the decomposition level, to avoid "overkill". Through calculation, the new threshold selection formula is expressed as

$$
\delta=\frac{\sigma \sqrt{2 \ln (n)}}{\ln \left(e+j^{2}-j\right)}
$$

Where $j$ is the number of layers of wavelet decomposition? By the formula (9) shows that the threshold changes are consistent with the conclusions of the experiments prove.

\subsection{Correlation Calculation of Wavelet Coefficients}

After wavelet decomposition still has some relevance are known, the combination of image noise, including a small amount of noise and uncorrelated noise characteristics, using the correlation calculation auxiliary de-noising, speaking to a small amount of noise removal.

Paper [13] proposed a classic denoising method (SSNF), the basic idea is to use the adjacent scale wavelet coefficients de-noising, and thus we have

$$
\begin{aligned}
& \operatorname{NCorr}_{L}(j, m, n)=\operatorname{Corr}_{L}(j, m, n) \sqrt{\frac{P_{w}(j)}{P_{c o r r}(j)}} \\
& \left|c_{j}(m, n)\right|=\min \left\{\left|\overline{\operatorname{NCorr}_{L}(j, m, n)}\right|\right\} \\
& \left|\overline{\operatorname{NCorr}_{L}(j, m, n)}\right|>k\left|c_{j}(m, n)\right|
\end{aligned}
$$

Where $\operatorname{corr}_{L}(j, m, n)=\prod_{i=0}^{L-1} w_{(m, n)}^{(i+j)}$ is coefficients of correlation, $p_{w}(j)$ is $j$ layer of wavelet coefficients, $P_{c o r r}(j)$ is $j$-layer energy of the correlation coefficient, $w_{(m, n)}^{(j)}$ is wavelet coefficient $j$ scale and coordinate $(m, n), L$ is the calculation of the product of the number of scale, $\mathrm{k}$ is a constant greater than zero.

Due to the correlation de-noising auxiliary de-noising, without the need for complex and a lot of computing, in order to simplify the range of the correlation calculation, correlation concept of the field is set to a size that the center of the window, and the calculated narrow area. By the correlation calculation to determine this coefficient and the coefficient around the center, and to determine whether it is the noise factor.

Related to the amount of the defined area $S(j, m, n)$ is denoted by $S(j, m, n)$, where $w_{(m, n)}^{(j)}$ for position $(m, n)$ scale wavelet coefficients, $j=1,2,3 \cdots \cdots$, According to the formula (11) and formula (12), we obtained

$$
\left|\overline{\operatorname{Norr}_{L}(j, m, n)}\right|>k \min \left\{\left|\overline{\operatorname{Norr}_{L}(j, m, n)}\right|\right\}
$$

Simplified as 


$$
C(m, n)=\frac{|w(m, n)|}{\min \left\{\left|w_{(m, n)}^{1}\right|, \cdots,\left|w_{(m, n)}^{j}\right|\right\}}
$$

When $\gamma \geq C(m, n) \geq 1$, wavelet coefficients' having a strong correlation and it is image signal; Otherwise, the wavelet coefficients of correlation is weak or irrelevant, and this wavelet coefficients representative of an image noise.

The selection of $\gamma$ here is very important, if it is close to 1 , the correlation estimate is too strict, if the value is large, easy to make noise Figure selected. The wavelet transform adaptive denoising algorithm flowchart is shown in Figure 1.

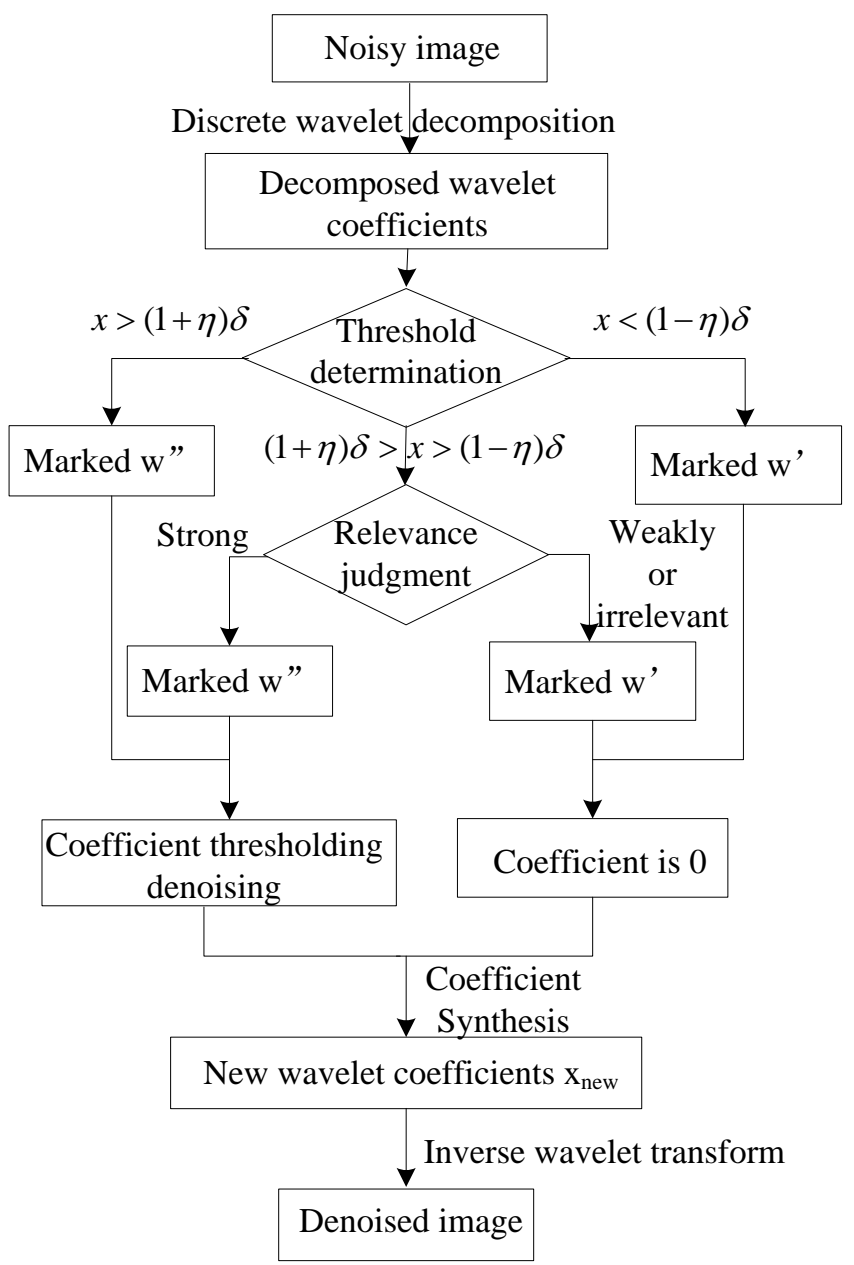

Figure 1. The Wavelet Transform Adaptive Denoising Algorithm Flowchart

\section{Experimental Results and Analysis}

The simulation software applications matlab2008a, conducted a number of simulation experiments, and the result is compared select wavelet bior3.7.

We perform our experiments on an infrared image [14]. Noisy images with different noise levels are generated by adding Gaussian white noise to the original noise-free images. 
Simulation experiments are performed using Matlab2008a. We use the adaptive threshold algorithm into image denoising with infrared image. We assume added additive noise is modeled by an I.I.D. Gaussian with zero mean and variance $\sigma^{2}$. Through experiments, we find that use the wavelet pair (sym12, coif4) which haves similar properties is better than others.

The experimental test images use in the 128-level grayscale image. Figure 2(a) shows the original image, Figure 2 (b) shows the image after adding Guassian noise, Figure 2(c) shows the effect of the soft threshold denoising, Figure 2(d) is the result by using the hard threshold denoising algorithm, Figure 2(e) is the result by using the compromise threshold denoising algorithm, Figure 2(f) is the result by using the proposed adaptive compromise threshold denoising algorithm. From the effects of several figures can be seen, in the case of adding random noise, using noise threshold of the proposed hybrid algorithm obtained by denoising the image clearly than the former effect has to be excellent.

Then add another Gaussian noise in the original image, the result as shown in Figure 3(a) and Figure 3(f). Figure 3(c) and Figure 3(e) is obtained by using the traditional means of noise reduction effect diagram. Figure 3(e) is the results by the proposed algorithm. Pieces can be seen from the results, in the case of added Gaussian noise, the noise threshold for the use of this adaptive algorithm are the denoised image is clearly better than the former effect has to be. Through a series of experiments, we can conclude that the proposed thresholdbased denoising adaptive denoising digital image regardless of the random noise or Gaussian noise has a better denoising effect.

The performance of the denoising algorithm is measured by the peak-signal-to-noise ratio (PSNR). The PSNR is defined as.

$$
P S N R=-\log _{10} \frac{\sum_{i, j}(B(i, j)-A(i, j))^{2}}{n^{2} \times 255^{2}}
$$

$\mathrm{B}$ is the denoised image and $\mathrm{A}$ is the noise-free image. To smooth out random fluctuation of the results, the same experiment is run 30 times and the average value of the PSNR is reported. We compare our results to other published results.

All the denoising results are shown in Table 1 which shows that the adaptive threshold provides the excellent denoising performance for the infrared image. Table 1 shows that the improved compromise threshold de-noising effect was significantly higher than the compromise threshold de-noising and soft and hard threshold de-noising, improve the edges of the image detail is lost in the denoising and image blur.

Table 1. PSNR(dB) Result for Several Denosing Algorithm

\begin{tabular}{|l|c|c|}
\hline & Simulation1 & Simulation2 \\
\hline Simulation parameters & $\alpha=25 \eta=0.2 \gamma=2$ & $\alpha=20 \eta=0.2 \gamma=2$ \\
\hline Noisy image & 22.5014 & 22.1030 \\
\hline Soft threshold & 25.1584 & 24.9731 \\
\hline Hard threshold & 24.0849 & 23.7578 \\
\hline Compromise threshold & 25.2825 & 25.1249 \\
\hline Improved Adaptive threshold & 25.4515 & 25.3448 \\
\hline
\end{tabular}




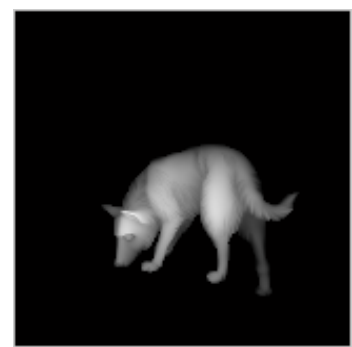

a) Original image

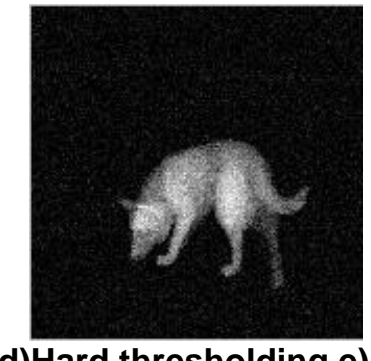

d)Hard thresholding e)

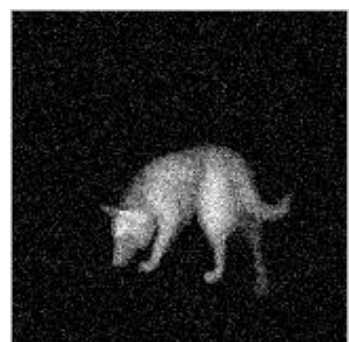

b) Noisy image

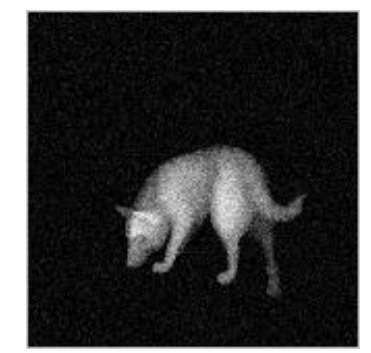

Compromise threshold

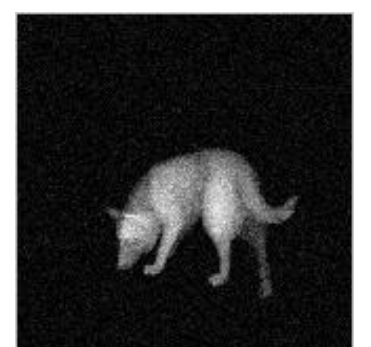

c) Soft thresholding denoising

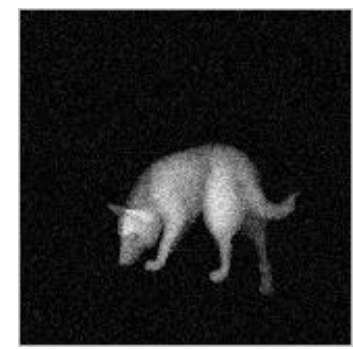

f) Adaptive denoising algorithm

\section{Figure 2. Results of Simulation 1}

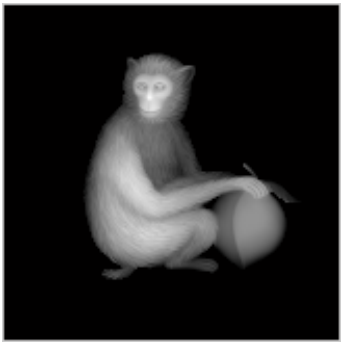

a) Original image

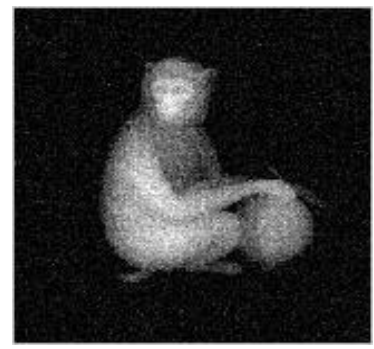

d)Hard thresholding

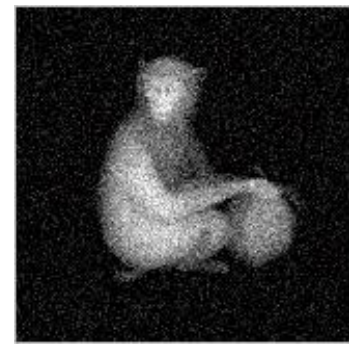

b) Noisy image denoising

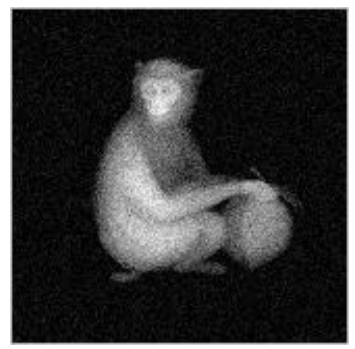

e) Compromise threshold

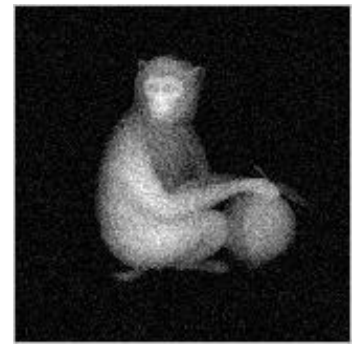

c) Soft thresholding

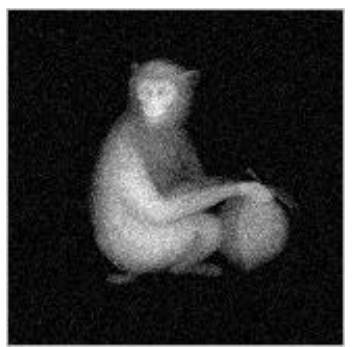

f) Adaptive denoising algorithm

Figure 3. Results of Simulation 2

\section{Conclusion}

This article studies the traditional hard threshold, soft threshold as well as the trade-off threshold de-noising method. On this basis put forward improved compromise threshold value 
denoising algorithm with coefficients relevance judgment, to increase the self-adaptive, more accurate removal of noise. A large number of experiments on soft threshold, hard threshold, and the compromise threshold are done to evaluate de-noising peak signal-to-noise ratio. Adaptive relevance threshold de-noising algorithm analysis and comparison of results show that adaptive relevant threshold de-noising algorithm more effective.

In the denoising process in order to achieve better denoising effect, the system takes more time to pay gray image; the other color digital image processing has not been a good result. Therefore, the authors will focus on late goals and improve the efficiency of color image denoising.

\section{Acknowledgments}

This work is partially supported by .scientific research fund of Heilongjiang Province Education Department, China (Grant No. 12521113). The authors also gratefully acknowledge the helpful comments and suggestions of the reviewers, which have improved the presentation.

\section{References}

[1] Y. S. Xu, J. B. Weaver, D. M. Healy and J. Lu, "Wavelet transform domain filters: a spatially selective noise filtration technique", IEEE Transactions on Image Processing, vol. 6 no.3, (1994), pp. 747 -758.

[2] M. Elad and A..M. Bruckstein, "A generalized uncertainty principle and sparse representation in pairs of bases", IEEE Transactions on Inform. Theory, vol. 48, no. 9, (2002), pp. 2558-2567.

[3] H. Liu, L. P. Zhai and Y. Gao, "Image Compression based on Biorthogonal wavelet transform", Proceeding of ISCIT (2005) December 10-12, pp. 578-581, Beijing.

[4] D. L. Donoho, "De-noising by soft- thresholding", IEEE Trans Inform Theory, vol. 3, (1995), pp. 613-627.

[5] J. Portilla, V. Strela, M. J. Wainwright and E. P. Simoncelli, "Image denoising using scale mixtures of Gaussian in the wavelet domain”, IEEE Trans. Image Process, vol. 12, no. 11, (2003), pp. 1338-1351

[6] L. Yingchun, S. Jiping and F. Xingiian, "Infrared image denoising based on wavelet transform", Laser and Infrared, vol. 10, no. 36, (2006), pp. 988-991.

[7] D. L. Donoho and I. M. Johnstone, "Adapting to unknown smoothness via wavelet shrinkage", J. Amer. Statist. Associated, vol. 90, (1995), pp. 1200-1224.

[8] S. G. Chang, B. Yu and M. Vetterli, "Adaptive wavelet thresholding for image denoising and compression", IEEE Trans. Image Processing, vol. 9, (2000), pp. 1532-1546.

[9] D. Donoho and M. Elad, "Optimally sparse representation in general (nonorthogonal) dictionaries via 11 minimization", Proceeding of National Academy of Science, (2003), pp. 2197-2202, USA.

[10] M. A. T. Figueiredo, "Adaptive sparseness for supervised learning", IEEE Trans. Pattern Anal. Machine Intell., vol. 25, no. 9, (2003), pp. 1150-1159.

[11] L. Sendur and I.W. Selesnick, "Bivariate shrinkage with local variance estimation", IEEE Lett. Signal Process, vol. 9, no. 12, (2002), pp. 438-441.

[12] X. Li and M.T. Orchard, "Spatially adaptive image denoising under over complete expansion", Proceedings of the IEEE International Conference on Image Processing, vol. 3, (2000) November 12-15, pp. 300-303. Singapore.

[13] S.K. Mohideen, Dr. S.A. Perumal and N. Krishnan, "Image denoising multi-wavelet and threshold", Proceedings of the 2008 International Conference on Computing, Communication and Networking, vol. 1, (2008) August 3-7, pp. 1-5, St. Thomas U.S. Virgin Islands.

[14] Y. Huixian, W. Xusi and X. Pinghe, "Improved threshold scale wavelet associated infrared image denoising", Acta Automatica Sinica, vol. 10, (2011), pp.1167-1174. 